\title{
From Anatomic Standardization Analysis of Perfusion SPECT Data to Perfusion Pattern Modelling
}

\author{
Christophe Grova ${ }^{1,2}$, Pierre Jannin ${ }^{1}$, Irène Buvat ${ }^{3}$, Habib Benali ${ }^{3}$, \\ Jean-Yves Bansard ${ }^{4}$, Arnaud Biraben ${ }^{1}$, and Bernard Gibaud ${ }^{1}$ \\ 1 Laboratoire IDM, Faculté de Médecine, Université de Rennes 1, France \\ \{pierre.jannin, arnaud.biraben, bernard.gibaud\}@univ-rennes1.fr, \\ http://idm.univ-rennes1.fr \\ 2 Montreal Neurological Institute, McGill University Montreal and INRIA France \\ \{christophe.grova\}@mail.mcgill.ca \\ 3 INSERM U494, CHU Pitié Salpétrière, Paris \\ \{irene.buvat, habib.benali\}@imed.jussieu.fr \\ 4 LTSI, université de Rennes 1, France \\ $\{$ jean-yves.bansard $\}$ @univ-rennes1.fr
}

\begin{abstract}
Our objective being to model SPECT brain perfusion patterns, the purpose of this study was to characterize inter-individual functional variability among a population of SPECT data. From perfusion measurements performed on anatomically standardized SPECT, we proposed to use correspondence analysis and hierarchical clustering to explore the structure of statistical dependencies among these measurements. The method was applied to study two perfusion patterns, namely normal perfusion and ictal perfusion as observed in temporal lobe epilepsy.
\end{abstract}

\section{Introduction}

Modelling functional inter-individual variability of brain perfusion among a population of SPECT data sets is a key issue, to design and assess powerful statistical analysis and detection methods. In this study, we propose a method to characterize such variability, as a first necessary step toward perfusion pattern modelling. We already generated average models of brain perfusion from anatomic standardization analysis of SPECT data, as part of a methodology to provide realistic SPECT simulations for evaluation purposes [1].

Volume of Interest (VOI)-based anatomic standardization analysis has been widely used to study normal perfusion patterns in SPECT (e.g., 2[3]). To model a perfusion pattern, such methods relied on an a priori spatial model of brain anatomy described by VOIs definition. VOI-based analysis assumes that the perfusion patterns of interest are homogeneous within each VOI. The main perfusion models reported in the literature reflected only average perfusion characteristics (e.g., [2]). Principal component analysis have also been proposed to study functional variability in healthy subjects [3]4]. 
The purpose of this study was to explore the structure of statistical dependencies, inherent to a perfusion pattern as observed in a population of SPECT data, using correspondence analysis (CA) and hierarchical clustering (HC). This multivariate analysis was performed on VOI measurements deduced from an anatomic standardization analysis. Two perfusion patterns were studied, namely normal perfusion and ictal perfusion as observed in temporal lobe epilepsy.

\section{Material and Methods}

\subsection{Anatomic Standardization Analysis}

Spatial model of brain anatomy: VOIs selection was deduced from the anthropomorphic model of the head proposed by Zubal et al. 55. This model was established from a T1-weighted high-resolution 3D MRI of a healthy subject from which VOIs were hand drawn and labeled. To generate a spatial model of brain anatomy dedicated to the study of perfusion patterns in temporal epilepsy [6], we segmented some temporal and frontal structures and lateralized all VOIs of the Zubal phantom. Fifty four lateralized brain VOIs were thus generated.

Population of subjects, data acquisition and preprocessing: Two populations of subjects exhibiting characteristic perfusion patterns were considered. SPECT scans of 27 healthy subjects (12 men and 15 women, aged 20 to 56 years) were used to study normal perfusion. These data were kindly provided by Dr. Barnden from the Queen Elizabeth Hospital (Woodville, Australia). Ten epileptic subjects (six men and four women, aged 19 to 43 years) from the Rennes University Hospital (Rennes, France) were selected to derive a model of ictal perfusion. All showed a mesial temporal epilepsy. After clinical investigations including SPECT acquisitions, they all underwent curative surgery and are now seizure free, so that the pathological hemisphere was clearly identified for each patient. For the 27 healthy subjects, SPECT images were acquired with a three-head gamma camera equipped with ultra high-resolution parallel collimators (IRIX, Philips MS) after injection of $500 \mathrm{MBq}$ of ${ }^{99 m} \mathrm{Tc}-\mathrm{HMPAO}$ (120 projections over $360^{\circ}, 128 \times 128$ matrix, pixel size $\left.=3.59 \mathrm{~mm}\right)$. For the ten epileptic patients, the ictal SPECT images were acquired with a two-head DST-XL camera (GEMS) equipped with fan beam or parallel ultra high-resolution collimators, after injecting $740 \mathrm{MBq}$ of ${ }^{99 m} \mathrm{Tc}-\mathrm{HMPAO}$ at the onset of the seizure $\left(64\right.$ projections over $360^{\circ}, 128 \times 128$ matrix, pixel size $\left.=4.51 \mathrm{~mm}\right)$. All SPECT data were reconstructed using filtered backprojection with a ramp filter (Nyquist frequency cutoff). The reconstructed data were post-filtered with an $8 \mathrm{~mm}$ full width at half maximum $(F W H M)$ 3D Gaussian filter. The spatial resolution was $F W H M=12.2 \mathrm{~mm}$ for all reconstructed images. Assuming uniform attenuation in the head, first order Chang attenuation correction was performed. Scatter correction was only performed for the healthy subjects using the Jaszczak method, by subtracting the projections corresponding to a simultaneous acquired Compton window. 
Spatial normalization: The spatial normalization method proposed by Friston et al. [7. (implemented in SPM softwar€1) was used to spatially normalize SPECT data and VOIs in the anatomic referential provided by the SPM T1 template. A non linear transformation was estimated to spatially normalize the Zubal phantom MRI data, hence the VOIs, to this referential. Concerning SPECT spatial normalization, affine transformations were accounted for. All these geometric transformations allowed resampling of the SPECT data and VOIs in the same mean anatomic referential, i.e. the T1 SPM template. Qualitative visual verification of the spatial normalization step was performed by a neurosurgeon, by superimposing the SPECT, MRI and VOIs in the same mean anatomic referential (see figure 1). No significant deformation errors were visually detected.

Perfusion measurements and intensity normalization: For each VOI $j$ and each SPECT data set $i$, a perfusion measurement consisted in the estimation of the mean $x_{i j}$ SPECT intensity within the VOI. To remove the confounding effect of global inter-acquisition changes in SPECT, each measurement was intensity normalized so that the mean voxel count throughout the brain was about 50 counts/voxel.
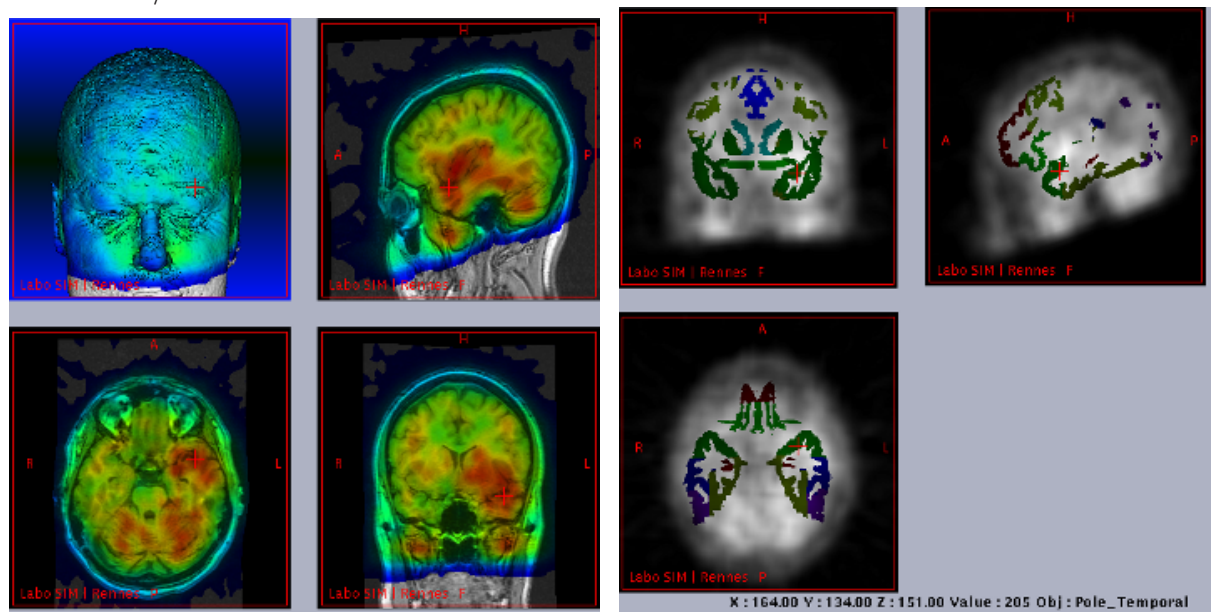

(a)

(b)

Fig. 1. Multimodal data fusion for anatomic standardization analysis: (a) ictal SPECT of an epileptic patient superimposed on its MRI, both being spatially normalized to the SPM T1 template (b) Frontal, temporal and some internal VOIs of the spatial model superimposed on the spatially normalized ictal SPECT of the same patient

\subsection{Perfusion Pattern Modelling}

We previously used those perfusion measurements to generate average normal and ictal perfusion models for SPECT simulation [1]. Now, the purpose of this

\footnotetext{
${ }^{1}$ Statistical Parametric Mapping (SPM) software : http://www.fil.ion.ucl.ac.uk/spm/
} 
study was to provide a more detailed analysis of the underlying distribution of such perfusion measurements. We explored functional variability by analyzing the structure of statistical dependencies between perfusion measurements. Considering the VOIs and the SPECT data sets as two qualitative variables, we chose to apply correspondence analysis (CA) as a well-known method to study the statistical dependencies between modalities of two qualitative variables [8].

\subsection{Correspondence Analysis (CA)}

$\mathrm{CA}$ relies on the analysis of a contingency table $\mathbf{N}$, where each element $n_{i j}$ describes the number of observed individuals partitioned among the modalities of two qualitative criteria.

Generation of the contingency table: In our study, the two qualitative variables consisted of the anatomical entities, i.e. the VOIs, and the SPECT data sets. More precisely, to emphasize inter-hemispheric asymmetries during the analysis, we considered measurements from both hemispheres as two realizations of the same qualitative variable. The variable $V O I_{\text {nonlat }}$ thus described the name of the non-lateralized VOIs of our spatial model, whereas the variable Subject $_{\text {Hem }}$ characterized the "name" of the measured hemisphere of each subject. We distinguished left versus right hemispheres for healthy subjects, and pathological versus healthy hemispheres for the epileptic patients. The variable Subject $_{H e m}$ was described by $m 1=2 \times 27=54$ (respectively $m 1=2 \times 10=20$ ) modalities for healthy subjects (respectively for epileptic patients). VOI nonlat was described by $m 2=54 / 2=27$ anatomical entities. A contingency table $\mathbf{N}$ was generated by assigning the corresponding measurement $x_{i^{\prime} j^{\prime}}$ (cf. 2.1 ) to each pair of Subject $_{\mathrm{Hem}}$ and $V O I_{\text {nonlat }}$ modalities.

Basics of CA: From a contingency table $\mathbf{N}$ described by $m 1$ rows and $m 2$ columns, row profiles were obtained by normalization of each row by the row sum, as follows:

$$
D_{1}^{-1} \mathbf{N} \text { with } D_{1}=\left(\begin{array}{cccc}
n_{1} & & & 0 \\
& n_{2} & & \\
& & \ddots & \\
0 & & & n_{m 1 .} .
\end{array}\right) \text { and } n_{i .}=\sum_{j=1}^{m 2} n_{i j}
$$

CA then consisted of a non-centered principal component analysis (PCA) applied to the row profiles table $D_{1}^{-1} \mathbf{N}$, using the metric $n D_{2}^{-1}$ and the weights $D_{1} / n$ (where by definition: $n=\sum_{i=1}^{m 1} \sum_{j=1}^{m 2} n_{i j}$ ) [8]. We considered here only the row profiles CA, but a similar analysis may be performed on column profiles and both analysis are dual. A row profile was then considered as an individual (i.e. a modality of Subject $_{H e m}$ ) described by $\mathrm{m} 2$ variables (i.e. modalities $V O I_{\text {nonlat }}$ ). The metric used to compare two row profiles actually consisted of a $\chi^{2}$ distance, a well-known similarity measure of statistical dependence. Such metric allows CA to compare the shapes of row profiles, and thus to provide an accurate analysis of the underlying structure of statistical dependencies among modalities of $V O I_{\text {nonlat }}$ and Subject Hem. $_{\text {. }}$. 


\subsection{Graphical Representations and Ascending Hierarchical Clustering}

A threshold on the decreasing eigenvalues resulting from CA allowed us to select the sub-space containing the most meaningful information. Variables or individuals were then represented by their projection on the first principal components. The center of principal axes represents the center of mass of the cloud: for complete statistical independence of both qualitative variables, all variables (or individuals) would be projected on this point. As a $\chi^{2}$ metric was used, points considered as neighbors and distant from the center of mass in such sub-space were interpreted as statistically dependent.

Graphical representation of the projections may be limited when the dimension of the selected sub-space exceeds 3 . We thus used an ascending hierarchical clustering (HC) analysis in such a sub-space to help interpretations. Clustering was achieved using the same $\chi^{2}$ metric used for CA, and the method of mutual neighbors was used to identify the clusters [8]. All statistical analysis were performed using BILOGINSERM software (C1979-1987-1993, INSERM, France).

\section{Results}

Perfusion measurements CA within healthy subjects: Projection of the variables on the first two principal axes is shown on figure 2 (a). The first three principal components were selected in order to recover $49 \%$ of global inertia. $\mathrm{HC}$ within such sub-space allowed us to distinguish three groups of anatomical structures, namely internal structures (Caudate Nucleus, Thalamus, Basal Ganglia and Lenticular Nucleus), mesio-temporal structures (Amygdala, Hippocampus and Temporal-Mesial + Cerebellum) and remaining cortex. Within each of these groups, perfusion measurements may then be considered as statistically dependent. Concerning the analysis of the individuals in this sub-space, a slight discrimination between the gender of the subjects was observed (results not shown).

Ictal perfusion measurements CA within epileptic subjects: The first two principal components were considered sufficient to extract main information of the CA of epileptic subjects (70.9\% of global inertia). Figure 2 (b) shows the projection of the variables on the first two principal axes. Similarly to healthy subjects $\mathrm{CA}, \mathrm{HC}$ within the selected sub-space allowed us to distinguish three groups corresponding to internal structures, temporal structures and remaining cortex. Some differences between normal and epileptic subjects were nevertheless observed: the VOI "Lenticular Nucleus" belonging to the group of internal structures for healthy subjects CA, was assigned to the group of temporal structures. The VOI "Temporal-Pole" classified in the remaining cortex for the healthy subjects, appeared as one of the most relevant structures describing the temporal structures group in the pathologic subjects. Finally, within the temporal structure group, two levels could be distinguished, namely: 


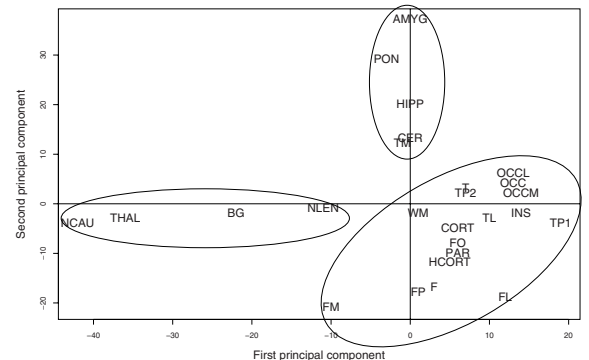

(a)

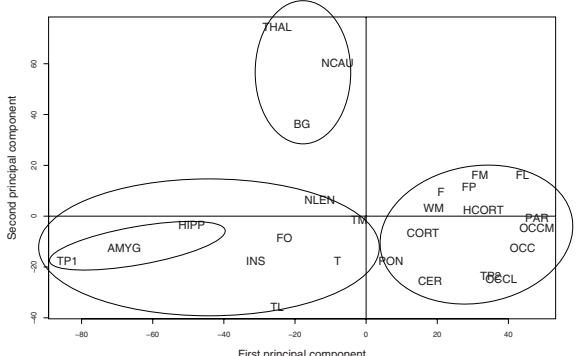

(b)

Fig. 2. Projection of the variables $\left(V O I_{\text {nonlat }}\right)$ on the first two principal components for CA of healthy subjects (a) and of epileptic patients (b). Ellipses were drawn from the HC results. Anatomical structures notations: CORT: Cortex, HCORT: Healthy Cortex, WM: White Matter, AMYG: Amygdala, HIPP: Hippocampus, NCAU: Caudate Nucleus, BG: Basal Ganglia, NLEN: Lenticular Nucleus, THAL: Thalamus, INS: Insula, T: Temporal, TP1: Temporal Pole, TM: Temporal Mesial, TL: Temporal Lateral, TP2: Temporal Posterior, F: Frontal, FP: Frontal Pole, FO: Frontal Orbit, FM: Frontal Mesial, FL: Frontal Lateral, OCC: Occipital, OCCM: Occipital Mesial, OCCL: Occipital Lateral, PAR: Parietal, CER: Cerebellum, PON: Pons

mesio-temporal structures (i.e., Amygdala, Hippocampus and Temporal-Pole) and surrounding temporal structures (i.e., Insula, Temporal, Temporal-Mesial, Temporal-Lateral, Frontal-Orbit and Lenticular Nucleus). The VOIs "TemporalPole" and "Lenticular-Nucleus" seem thus very relevant to describe the ictal perfusion pattern. The dual representation showing the projection of individuals on the first two principal axes showed a clear relevant discrimination between pathological and healthy explored hemispheres (results not shown).

\section{Discussion}

From SPECT perfusion measurements deduced from a VOI-based anatomic standardization approach, we explored the statistical dependencies between measurements using CA followed by $\mathrm{HC}$ on the selected sub-space. The method was used to study normal perfusion and ictal perfusion as seen in mesio-temporal epilepsy. Structure of dependencies between perfusion measurements was found, suggesting that $\mathrm{CA}$ is a promising approach to model functional variability among a population of SPECT data.

Considering the 27 healthy subjects, three groups of structures, namely internal structures, mesio-temporal structures and remaining cortex, were exhibited as relevant to describe the structure of statistical dependencies among the perfusion pattern. Concerning the individuals, a gender effect on the perfusion measurements was exhibited. Our results agree with other studies of normal SPECT perfusion, that showed significant effects of various parameters of the population [2,3] (e.g. age, gender, laterality). Similar clusters have been reported by Pagani et al. [3], who used PCA to analyze VOI-based perfusion measure- 
ments. To explain $81 \%$ of the variance, they extracted 12 components, whereas 7 components were sufficient to explain a similar percentage of global inertia on our experience. Differences between Pagani's results and our results may partly be explained by different acquisition and post-processing protocols and by the population of subjects. We studied less data than Pagani et al. and proved that the ages of our studied subjects were not uniformly distributed. Such results outline the complexity of the structure of normal SPECT perfusion but give the main trends of its structure. Special attention must be paid regarding the selection of the studied population of healthy subjects. Epileptic patients selected for this study were known to exhibit a characteristic ictal perfusion pattern [6], and chosen for our perfusion modelling purpose. To take into account such a priori on the spatial structure of the pattern, we also tuned our spatial model by re-segmenting some VOIs of the Zubal phantom. Due to extreme perfusion changes caused by epileptic seizure, it was expected that CA discriminates between pathological and healthy hemispheres. Moreover, CA and HC results showed that the global structure of statistical dependencies between ictal and normal perfusion measurements was similar, except for the VOIs "TemporalPole" and "Lenticular Nucleus", which therefore seemed relevant to characterize such ictal perfusion pattern. The understanding of the behavior of the temporal pole in mesio-temporal epilepsy is still an interesting open question [9].

Our results should be interpreted with cautious, especially because of the limited number of SPECT data studied. Indeed, it is not obvious to distinguish which part of the results is actually due to physiological or physiopathological aspects, and which is generated from our measurement methodology. Several explanations may be proposed for the observed phenomena. The dependencies between perfusion measurements may be caused by spatial correlations due to the limits of our inter-individual analysis method and to the bias of SPECT perfusion measurements caused by acquisition and preprocessing methods. As spatial resolution might greatly affect the range of statistical dependency between neighboring regions, we used processing protocols ensuring that the spatial resolution in the reconstructed images was similar (i.e. $12.2 \mathrm{~mm}$ ). We can then reasonably assume that the differences in dependencies seen between the two groups are not due to differences in spatial resolution. Moreover, as CA and HC performed on healthy subjects before and after scatter correction lead to similar results (results not shown), we may assume that scatter does not affect the structure of statistical dependencies between regions. Partial volume effect due to SPECT limited spatial resolution is certainly one of the most influent effects, especially to explain statistical dependencies between small neighboring VOIs. A new partial volume effect correction method recently proposed to correct VOI-based PET measurements [10] may be an interesting approach for further investigations. By choosing CA, our approach differed from other reported studies using PCA 3, 4 as we explored dependencies between shapes of perfusion measurement distributions, whereas PCA relies more on intensity correlations. Both independently computed CA allowed us to exhibit such characteristic features or shapes within each population.

Our study showed preliminary results that could be used to provide more realistic perfusion models. An application may be to select only relevant principal 
components from a studied population CA, to create a distribution of realistic activity maps for SPECT simulations. Such simulated data could be dedicated to the evaluation of SPECT detection methods as for instance inter-hemispheric asymmetries detection [1] or ictal SPECT/inter-ictal SPECT subtraction.

Acknowledgments. This work was partly supported by a grant from the "Conseil Régional de Bretagne". C. Grova is now funded by the "Institut National de Recherche en Informatique et Automatique" (INRIA, France). We also would like to thank Dr. Barnden (Woodville, Australia) for providing normal SPECT data.

\section{References}

1. C. Grova, P. Jannin, A. Biraben, I. Buvat, H. Benali, A.M. Bernard, J.M. Scarabin, and B. Gibaud. Validation of MRI/SPECT similarity-based registration methods using realistic simulations of normal and pathological SPECT data. In CARS 2002, Paris, pages 450-455, Berlin, Heidelberg, 2002. Springer-Verlag.

2. K. Van Laere, J. Versijpt, K. Audenauert, M. Koole, I. Goethals, E. Achten, and R. Dierckx. 99mTc-ECD brain perfusion SPET: Variability, asymmetry and effect of age and gender in healthy adults. EJNM, 28(7):873-887, 2001.

3. M. Pagani, D. Salmaso, C. Jonsson, R. Hatherly, H. Jacobsson, S.A. Larsson, and A. Wägner. Regional cerebral blood flow assessment by principal component analysis and 99mTc-HMPAO SPET in healthy subjects at rest: Normal distribution and effect of age and gender. EJNM, 29(1):67-75, 2002.

4. A. Houston, P. Kemp, and M. Macleod. A method for assessing the significance of abnormalities in HMPAO brain SPECT images. JNM, 35(2):239-244, 1994.

5. I.G. Zubal, C.R. Harrell, E.O. Smith, Z. Rattner, G.R. Gindi, and P.B. Hoffer. Computerized three-dimensional segmented human anatomy. Med. Phys., 21(2):299-302, 1994.

6. S.S. Ho, S.F. Berkovic, W.J. McKay, R.M. Kalnins, and P.F. Bladin. Temporal lobe epilepsy subtypes: Differential patterns of cerebral perfusion on ictal SPECT. Epilepsia, 37(8):788-795, 1996.

7. K.J. Friston, J. Ashburner, J.B. Poline, C.D. Frith, J.D. Heather, and R.S.J. Frackowiak. Spatial registration and normalization of images. HBM, 2:165-189, 1995.

8. L. Lebart, A. Morineau, and K. M. Warwick. Multivariate Descriptive Statistical Analysis. Wiley series in probability and mathematical statistics, New York, 1984.

9. P. Ryvlin, P. Kahane, A. Arzimanoglou, and F. Anderman (Editeurs). Epileptic Disorders : Temporal Pole and Mesiotemporal Epilepsy, volume 4 (Suppl. 1). John Libbey Eurotext, 2002.

10. V. Frouin, C. Comtat, A. Reilhac, and M-C. Grégoire. Correction of partial-volume effect for PET striatal imaging: Fast implementation and study of robustness. JNM, 43(12):1715-1726, 2002.

11. B. Aubert-Broche, C. Grova, P. Jannin, I. Buvat, H. Benali, and B. Gibaud. Detection of inter-hemispheric asymmetries of brain perfusion in SPECT. Phys. Med. Biol., 48(11):1505-1517, 2003. 